\title{
VALIDAÇÃo DA METOdOLOGIA PARA DETERMinAÇÃo SIMULTÂNEA, POR CLAE, DE COLESTEROL E ÓXIDOS DE COLESTEROL EM PRODUTOS CÁRNEOS PROCESSADOS ${ }^{1}$
}

\author{
Sueli Regina BAGGIO², Neura BRAGAGNOLO ${ }^{2, *}$
}

\begin{abstract}
RESUMO
No presente trabalho foi validado um método para a determinação simultânea de colesterol e óxidos de colesterol em produtos cárneos processados, por cromatografia líquida de alta eficiência (CLAE), utilizando detectores por conjunto de diodos e índice de refração. Inicialmente foram testados cinco métodos e oito condições cromatográficas. O método selecionado foi de acordo com SANDER et al. [25], o qual apresenta as seguintes etapas: extração dos lipídios, saponificação a frio e extração da matéria insaponificável. As condições cromatográficas estabelecidas foram: coluna Nova Pak CN HP ( $300 \times 3,9 \mathrm{~mm}, 4 \mu \mathrm{m})$; temperatura da coluna $32^{\circ} \mathrm{C}$; fase móvel de hexano/ isopropanol (96+4) com vazão de $1,0 \mathrm{~mL} / \mathrm{min}$, detectores por conjunto de diodos fixado a $210 \mathrm{~nm}$ e índice de refração. O método foi validado através da recuperação, repetibilidade, limite de detecção, limite de quantificação e comparação dos resultados obtidos pelos dois detectores. A identificação do colesterol e dos óxidos de colesterol foi feita por comparação do tempo de retenção do padrão e o da amostra, espectros de absorvância e co-cromatografia, e a confirmação por espectrometria de massas. Nas condições cromatográficas utilizadas, foram separados o colesterol e os seguintes óxidos de colesterol: colesta-4,6-dien-3-ona, 20 $\alpha$-hidroxicolesterol, 25-

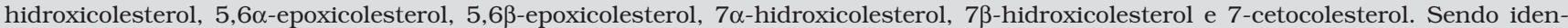
tificados e confirmados nas amostras analisadas o colesterol, o 7-cetocolesterol e o 5,6 $\beta$-epoxicolesterol. O colesterol e o 5,6 $\beta$ epoxicolesterol foram quantificados pelo detector por índice de refração e o 7-cetocolesterol pelo detector por conjunto de diodos.

Palavras-chave: colesterol; óxidos de colesterol; CLAE; produtos cárneos processados.
\end{abstract}

\section{SUMMARY}

VALIDATION OF HPLC METHODOLOGY FOR THE SIMULTANEOUS DETERMINATION OF CHOLESTEROL AND CHOLESTEROL OXIDES IN PROCESSED MEAT PRODUCTS. This study aimed at validating a method for the simultaneous determination of cholesterol and cholesterol oxides in processed meat products by high performance liquid chromatography (HPLC), using a diode array and a refractive index detectors. Initially five methods and eight chromatographic conditions were tested. The selected method was that of SANDER et al. [25], which presents the following steps: lipid extraction, cold saponification and extraction of non-saponifyable material. The chromatographic conditions established were: Nova Pak CN HP column $(300 \times 3.9 \mathrm{~mm}, 4 \mu \mathrm{m})$; column temperature of $32^{\circ} \mathrm{C}$; mobile phase of hexane/isopropanol $(96+4)$ with a flow rate of $1.0 \mathrm{~mL} / \mathrm{min}$, diode array detector fixed at $210 \mathrm{~nm}$ and a refractive index detector. The method was validated by way of the recuperation, repeatability, detection limits, quantification limits and comparison of the results obtained with the two detectors. The identification of the cholesterol and cholesterol oxides was by comparison of the retention times of the samples with those of the standards, absorbance spectra and co-chromatography, with confirmation by mass spectroscopy. Under the chromatographic conditions used, cholesterol and the following cholesterol oxides were separated: colesta-4,6-dien-3-one, 20 $\alpha$ hidroxycholesterol, 25-hydroxycholesterol, 5,6 $\alpha$-epoxicholesterol, 5,6ß-epoxycholesterol, $7 \alpha$-hidroxycholesterol, $7 \beta$-hidroxycholesterol and 7-cetocholesterol. Cholesterol, 7-cetocholesterol and 5,6 $\beta$-epoxycholesterol were confirmed in the samples analyzed. Cholesterol and 5,63-epoxycholesterol were quantified using the refractive index detector and 7-cetocholesterol using the diode array detector. Keywords: cholesterol; cholesterol oxides; HPLC; processed meat products.

\section{1 - INTRODUÇÃO}

O colesterol é um importante constituinte dos produtos de origem animal, pois apresenta funções importantes no organismo humano. Está presente em todas as membranas celulares, é a chave intermediária na síntese de ácidos biliares, hormônios e participa da sintese da vitamina $\mathrm{D}_{3}$.

O colesterol é um lipídio insaturado, susceptível à oxidação, levando à formação de vários produtos de oxidação. A autoxidação do colesterol pode ocorrer sob várias condições, como a exposição do mesmo ao ar, a temperaturas elevadas, iniciadores de radicais livres, luz ou à combinação destes [28]. Os principais óxidos de colesterol encontrados em alimentos são: 25-hidroxicolesterol,

1. Recebido para publicação em 28/09/2002. Aceito para publicação em 30/12/2003 (000981).

2. Faculdade de Engenharia de Alimentos, UNICAMP, Departamento de Ciência de Alimentos, Cx. Postal 6121, CEP 13083-970, Campinas-SP. E-mail: neura@fea.unicamp.br

*A quem a correspondência deve ser enviada. colestan-3 $\beta-5 \alpha-6 \beta$-triol, $\quad 5,6 \alpha$-epoxicolesterol, $\quad 5,6 \beta$ epoxicolesterol, $7 \alpha$-hidroxicolesterol, $7 \beta$-hidroxicolesterol e 7-cetocolesterol [5].

Os produtos da oxidação do colesterol apresentam possivelmente um papel mais importante no desenvolvimento de placas ateroscleróticas do que o próprio colesterol [9, 10, 22], bem como outros efeitos biológicos indesejáveis como citotoxicidade, carcinogenicidade, mutagenicidade, alterações nas propriedades das membranas celulares e inibição da atividade da enzima HMGCoA (3-hidroxi-3-metil-glutaril-CoA) redutase [8].

Há uma grande variedade de metodologias para a determinação de óxidos de colesterol em produtos alimentícios, no entanto, é observada discrepância nos resultados. A quantificação é também um grande desafio, especialmente em alimentos contendo baixos niveis destes compostos, pois o isolamento dos mesmos é freqüentemente dificultado pela quantidade de interferentes, tais como: colesterol, triacilgliceróis e fosfolipídios [11]. Requer uma metodologia analítica branda para evitar a formação de artefatos, uma vez que a presença do 
ar, luz, solventes contendo peróxidos ou tratamento térmico podem promover a formação de óxidos [24].

A separação dos produtos da oxidação do colesterol foi inicialmente realizada por cromatografia em camada delgada (CCD) [1]. Esta técnica cromatográfica separa alguns compostos como os hidroxicolesteróis, mas não os hidroperoxicolesteróis [30]. Além disso, há maior exposição das amostras à oxidação como resultado da grande área de superfície e da menor velocidade de eluição dos compostos [4].

A maioria dos trabalhos na literatura tem utilizado cromatografia gasosa (CG) para separar os óxidos de colesterol $[5,12,15,17,18,19,20,25,31]$. Embora seja eficiente para separar alguns produtos da oxidação do colesterol, a CG pode também destruir termicamente o colesterol e os hidroperóxidos [6, 31]. A cromatografia gasosa com espectrometria de massas (CG-MS) é também uma técnica particularmente eficaz para a confirmação dos óxidos de colesterol [16, 20, 24]. Um outro método cromatográfico bastante empregado é a cromatografia líquida de alta eficiência (CLAE) [1, 21], porém, a determinação simultânea dos óxidos de colesterol por CLAE é uma tarefa difícil, porque alguns compostos apresentam diferentes comprimentos de onda de absorção na luz ultravioleta (UV), enquanto outros óxidos de importância

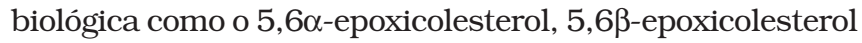
e o colestan-3 $\beta-5 \alpha-6 \beta$-triol não possuem características adequadas de absorção na luz UV [1, 4, 29]. Alguns autores têm utilizado detector por índice de refração, embora tenha menos sensibilidade que o UV [3, 13].

Assim, o objetivo deste trabalho foi otimizar e validar uma metodologia por CLAE para a determinação simultânea de colesterol e óxidos de colesterol em produtos cárneos processados, utilizando detector por conjunto de diodos e detector por índice de refração.

\section{2 - MATERIAL E MÉTODOS}

\section{1 - Materiais}

Para a validação e otimização da metodologia, foram utilizadas amostras de produtos processados de carne de peru, sendo peito defumado, almôndegas, blanquete, hambúrguer, presunto, presunto defumado, roulé e salsicha. As amostras foram adquiridas em supermercados de Campinas e imediatamente analisadas. As amostras foram trituradas e homogeneizadas em multiprocessador até obtenção de uma massa homogênea. Alíquotas de $50 \mathrm{~g}$ foram utilizadas para análises em duplicata.

O colesterol e os óxidos de colesterol: colesta-4,6dien-3-ona, 20 $\alpha$-hidroxicolesterol, 25-hidroxicolesterol, 7cetocolesterol, 5,6 $\alpha$-epoxicolesterol, 5,6 6 -epoxicolesterol e $7 \beta$-hidroxicolesterol foram adquiridos da Sigma Chemical Company (St. Louis, EUA). O $7 \alpha$-hidroxicolesterol foi obtido da Steraloids Inc. (Wilton, EUA).

Todos os solventes usados para a extração e preparo da amostra foram grau P.A. e para análise cromatográfica foram grau cromatográfico, os quais foram filtrados e degaseificados em ultra-som antes do uso.

\section{2 - Métodos}

Para a determinação simultânea de colesterol e óxidos de colesterol em produtos cárneos processados por cromatografia líquida de alta eficiência (CLAE), foram testados vários métodos de saponificação e extração da matéria insaponificável. Os métodos testados foram de acordo com SARANTINOS, O'DEA \& SINCLAIR [26], CHEN, CHIU \& CHEN [3], PARK \& ADIS [18], NOVELLI et al. [14] e SANDER et al. [25].

Foi utilizado um cromatógrafo líquido Schimadzu (Kioto, Japão) com sistema quaternário de solventes (LC-10ATVP), válvula "Rheodyne" com alça de amostragem de $20 \mu \mathrm{L}$, forno de coluna (CTO-10ASVP), dois detectores ligados em série: detector por conjunto de diodos (SPD-M10AVP) e detector por índice de refração (RID-10A), e "software" (CLASS-VP). Os espectros de absorvância foram tirados entre 200 e 400nm e os cromatogramas foram registrados a $210 \mathrm{~nm}$. Foram testadas várias condições cromatográficas, as quais estão relacionadas na Tabela 1 .

TABELA 1. Condições cromatográficas testadas

\begin{tabular}{|c|c|c|c|c|c|}
\hline Condição & Coluna & $\begin{array}{c}\text { Coluna de } \\
\text { Guarda }\end{array}$ & $\begin{array}{l}\text { Temp. da } \\
\text { coluna }\left({ }^{\circ} \mathrm{C}\right)\end{array}$ & $\begin{array}{l}\text { Fase } \\
\text { móvel }\end{array}$ & $\begin{array}{c}\text { Vazão } \\
\text { (mL/min) }\end{array}$ \\
\hline a & $\begin{array}{c}\text { Microsorb } \\
\text { C18 } \\
\text { (Rainin) } \\
\text { 100X4,6mm; } \\
3 \mu \mathrm{m}\end{array}$ & Sem & 35 & $\begin{array}{c}\text { acetonitrila/ } \\
\text { água } \\
90+10)\end{array}$ & 1,5 \\
\hline b & $\begin{array}{c}\text { Lichrospher } \\
100 \mathrm{RP} 18 \\
\text { 125x4mm; } \\
5 \mu \mathrm{m}\end{array}$ & $\begin{array}{c}\text { Lichrospher } \\
100 \mathrm{RP} 18 \\
4 \times 4 \mathrm{~mm} ; \\
5 \mu \mathrm{m}\end{array}$ & 35 & $\begin{array}{c}\text { acetonitrila/ } \\
\text { isopropanol } \\
(70+30)\end{array}$ & 0,8 \\
\hline c & $\begin{array}{c}\text { Lichrospher } \\
100 \mathrm{RP} 18 \\
\text { 125x4mm; } \\
5 \mu \mathrm{m}\end{array}$ & $\begin{array}{c}\text { Lichrospher } \\
100 \mathrm{RP} 18, \\
4 \times 4 \mathrm{~mm} ; \\
5 \mu \mathrm{m}\end{array}$ & 35 & $\begin{array}{c}\text { acetonitrila/ } \\
\text { isopropanol } \\
(82+18\end{array}$ & 1,2 \\
\hline d & $\begin{array}{c}\text { Lichrospher } \\
100 \mathrm{RP} 18 \\
250 \times 4 \mathrm{~mm} \\
5 \mu \mathrm{m}\end{array}$ & $\begin{array}{c}\text { Lichrospher } \\
100 \mathrm{RP} 18 \\
4 \times 4 \mathrm{~mm} ; \\
5 \mu \mathrm{m}\end{array}$ & 35 & $\begin{array}{c}\text { hexano/ } \\
\text { isopropanol } \\
(90+10)\end{array}$ & 1,5 \\
\hline e & $\begin{array}{c}\text { Lichrospher } \\
100 \mathrm{RP} 18 \\
250 \times 4 \mathrm{~mm} ; \\
5 \mu \mathrm{m}\end{array}$ & $\begin{array}{c}\text { Lichrospher } \\
100 \mathrm{RP} 18 \\
4 \times 4 \mathrm{~mm} ; \\
5 \mu \mathrm{m}\end{array}$ & 35 & $\begin{array}{c}\text { hexano/ } \\
\text { isopropanol } \\
(90+10)\end{array}$ & 1,0 \\
\hline$f$ & $\begin{array}{c}\text { Lichrospher } \\
100 \mathrm{RP} 18 \\
250 \times 4 \mathrm{~mm} \\
5 \mu \mathrm{m}\end{array}$ & $\begin{array}{c}\text { Lichrospher } \\
100 \mathrm{RP} 18, \\
4 \times 4 \mathrm{~mm} ; \\
5 \mu \mathrm{m}\end{array}$ & 35 & $\begin{array}{c}\text { hexano/ } \\
\text { isopropanol } \\
(95+5)\end{array}$ & 1,0 \\
\hline $\mathbf{g}$ & $\begin{array}{c}\mu \text {-Porasil } \\
\text { (sílica) } \\
\text { 250X4,6mm; } \\
5 \mu \mathrm{m}\end{array}$ & Sem & 35 & $\begin{array}{c}\text { hexano/ } \\
\text { isopropanol } \\
(97+3)\end{array}$ & 1,0 \\
\hline h & $\begin{array}{c}\text { Nova Pak } \\
\text { CN HP } \\
300 \times 3,9 \mathrm{~mm} ; \\
4 \mu \mathrm{m}\end{array}$ & $\begin{array}{c}\text { Hypersil } \\
\text { BDS CN, } \\
7,5 \times 4,6 \mathrm{~mm} \text {; } \\
5 \mu \mathrm{m}\end{array}$ & 32,35 e 40 & $\begin{array}{c}\text { hexano/ } \\
\text { isopropanol } \\
(96+4)\end{array}$ & 1,0 \\
\hline
\end{tabular}

A confirmação da identidade de cada pico foi realizada por cromatografia gasosa com espectrometria de massas (CG-MS), usando um CG modelo HP 6890 acoplado a um MS HP 5973 (Palo Alto, USA). A temperatura de interface foi de $320^{\circ} \mathrm{C}$ e a energia de impacto de elétrons foi de $70 \mathrm{eV}$. Injetor splitless a $290^{\circ} \mathrm{C}$ e coluna 
capilar de sílica fundida HP-5MS (30m x 0,25mm; espessura do filme $0,25 \mu \mathrm{m}$ ) com temperatura programada: temperatura inicial $110^{\circ} \mathrm{C} / 2$ minutos, elevou-se para $235^{\circ} \mathrm{C}$ numa escala de $40^{\circ} \mathrm{C} /$ minuto, permanecendo nesta temperatura por 5 minutos. Após elevou-se a temperatura para $310^{\circ} \mathrm{C} / 5 \mathrm{~min}$ numa escala de $1^{\circ} \mathrm{C} / \mathrm{minu}-$ to. O fluxo do gás de arraste (hélio) foi de $0,7 \mathrm{~mL} / \mathrm{minuto}$. Os padrões de colesterol e de óxidos de colesterol e as amostras foram derivatizados de acordo com o método de SCHMARR, GROSS \& SHIBAMOTO [27].

A quantificação do colesterol e de seus produtos da oxidação foi feita por padronização externa. A curva padrão foi construída com concentrações variando de 0,5 a $2,22 \mathrm{mg} / \mathrm{mL}$ para colesterol e de 0,5 a $64,0 \mu \mathrm{g} / \mathrm{mL}$ para óxidos de colesterol.

A recuperação do método para produtos cárneos processados foi feita utilizando-se amostras de peito de peru defumado, adicionando-se $10 \mathrm{mg}$ de colesterol e $40 \mu \mathrm{g}$ de 7 -cetocolesterol e 5,63-epoxicolesterol.

O limite de detecção e o limite de quantificação para o colesterol e para os óxidos de colesterol foram calculados de acordo com CHAIRMAN et al. [2]. A média do desvio padrão de dez repetições multiplicado por três é igual ao limite de detecção, e multiplicado por dez é igual ao limite de quantificação.

\section{3 - RESULTADOS E DISCUSSÃO}

Para a determinação simultânea por CLAE de colesterol e óxidos de colesterol em produtos cárneos processados foi escolhido o método segundo SANDER et al. [25], que possui as seguintes etapas: extração dos lipídios de acordo com FOLCH, LESS \& STANLEY [7], saponificação a frio e extração da matéria insaponificável com éter etílico. O método está sumariamente descrito na Figura 1.

Tomar $50 \mathrm{~mL}$ do extrato lipídico e secar sob $\mathrm{N}_{2}$

$$
\downarrow
$$

Adicionar $10 \mathrm{~mL}$ de $\mathrm{KOH} 1 \mathrm{M}$ em metanol e agitar a temperatura de $20^{\circ} \mathrm{C}$

durante 20 horas

$$
\downarrow
$$

Extrair a matéria insaponificável com $3 \times 10 \mathrm{~mL}$ de éter etílico e lavar

com $5 \mathrm{~mL}$ de $\mathrm{KOH} 0,5 \mathrm{M}$ e $2 \times 5 \mathrm{~mL}$ de água destilada

$\downarrow$

Filtrar com $\mathrm{Na}_{2} \mathrm{SO}_{4}$ anidro e lavar com mais $10 \mathrm{~mL}$ de éter etílico

$\downarrow$
Secar sob $\mathrm{N}_{2}$
$\downarrow$
Dissolver em $2 \mathrm{~mL}$ de hexano/isopropanol (96+4)
$\downarrow$
CLAE

FIGURA 1. Fluxograma da determinação simultânea de colesterol e óxidos de colesterol de acordo com SANDER et al. [25].
Este método foi o que apresentou melhor resultado, permitindo a determinação simultânea de colesterol e óxidos de colesterol e a utilização de um menor volume de solventes. Nos métodos segundo CHEN, CHIU \& CHEN [3] e PARK \& ADDIS [18], que supostamente separa o colesterol dos óxidos de colesterol, nas condições testadas, isto não ocorreu, verificando-se que não havia a separação total do colesterol bem como dos óxidos de colesterol. Além disso, para a determinação do colesterol e dos óxidos de colesterol seria necessário duas injeções no CLAE, o que consumiria muito tempo. Uma outra inconveniência observada no método de acordo com PARK \& ADDIS [18], foi a pequena quantidade de amostra utilizada, dificultando a quantificação dos compostos da oxidação do colesterol, pois estes, quando formados, encontram-se em baixas concentrações. Nos métodos segundo SARANTINOS, D'ODEA \& SINCLAIR [26] e NOVELLI et al. [14], embora muito parecidos entre si, e, com o método escolhido, observou-se a formação de uma emulsão compacta dificultando a separação das fases. Uma outra desvantagem do método de acordo com NOVELLI et al. [14] foi o grande consumo de solventes.

TABELA 2. Ordem de eluição cromatográfica do colesterol e

\begin{tabular}{|c|c|c|}
\hline \multicolumn{3}{|l|}{ Ordem de eluição } \\
\hline Cromatográfica* & Nome sistemático & Nome comum \\
\hline 1 & Colest-5-eno-3 $\beta$-ol & colesterol \\
\hline 2 & Colesta-4,6-dien-3-ona & dieno \\
\hline 3 & Colest-5-eno-3 $3,20 \alpha$-diol & $20 \alpha$-hidroxicolesterol \\
\hline 4 & Colest -5-eno-3 $\beta, 25$-diol & 25- hidroxicolesterol \\
\hline 5 & 5,6- $\alpha$-epoxi- $5 \alpha$-cholestan- $3 \beta$-ol & $\alpha$-epoxicolesterol** \\
\hline 6 & 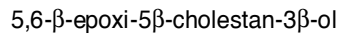 & $\beta$ - epoxicolesterol ** \\
\hline 7 & 3 $\beta$-hidroxicolest-5-eno-7-ona & 7-cetocolesterol \\
\hline 8 & Colesta-5-eno-3 $\beta, 7 \beta$-diol & $7 \beta$-hidroxicolesterol \\
\hline 9 & 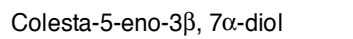 & $7 \alpha$-hidroxicolesterol \\
\hline
\end{tabular}
óxidos de colesterol

*De acordo com as Figuras 1 e 2.
$*$ *Somente no índice de refração.

Para a determinação simultânea por CLAE de colesterol e óxidos de colesterol, utilizando detectores por conjunto de diodos e índice de refração, a condição cromatográfica que apresentou melhor resultado, isto é, maior seletividade, resolução e eficiência foi usando uma coluna de guarda Hypersil BDS CN (7,5 x 4,6mm, $5 \mu \mathrm{m})$; coluna Nova Pak CN HP (300 x 3,9mm, $4 \mu \mathrm{m}$, Watters, USA); temperatura da coluna de $32^{\circ} \mathrm{C}$; fase móvel composta de hexano/isopropanol (96+4) com vazão de $1,0 \mathrm{~mL} / \mathrm{min}$. Nestas condições cromatográficas, conseguiu-se separar o colesterol e os óxidos de colesterol: colesta-4,6-dien-3-ona, 20 $\alpha$-hidroxicolesterol, 25hidroxicolesterol, 7-cetocolesterol, $7 \beta$-hidroxicolesterol e $7 \alpha$-hidroxicolesterol, pelo detector por conjunto de diodos e todos estes compostos mais o 5,6 $\alpha$ epoxicolesterol e o 5,6 $\beta$-epoxicolesterol, pelo detector por índice de refração.O colestan- $3 \beta, 5 \alpha, 6 \beta$-triol não foi detectado, mesmo em altas concentrações. O coleste- 
rol e os óxidos de colesterol determinados nas amostras analisadas pelo detector por índice de refração foram identificados através da comparação dos tempos de retenção do padrão e o da amostra e co-cromatografia, e, quando determinados pelo detector por conjunto de diodos, além dos parâmetros utilizados no índice de refração, os picos também foram identificados através dos espectros de absorvância (Figuras 2 a 5). A ordem de eluição cromatográfica do colesterol e seus óxidos encontra-se na Tabela 2. Nas amostras de produtos processados de carne de peru analisadas foram identificados o colesterol, o 7-cetocolesterol e o 5,6 3 epoxicolesterol, os quais foram confirmados por espectrometria de massa, cujos espectros de massas estão apresentados nas Figuras 6, 7 e 8.
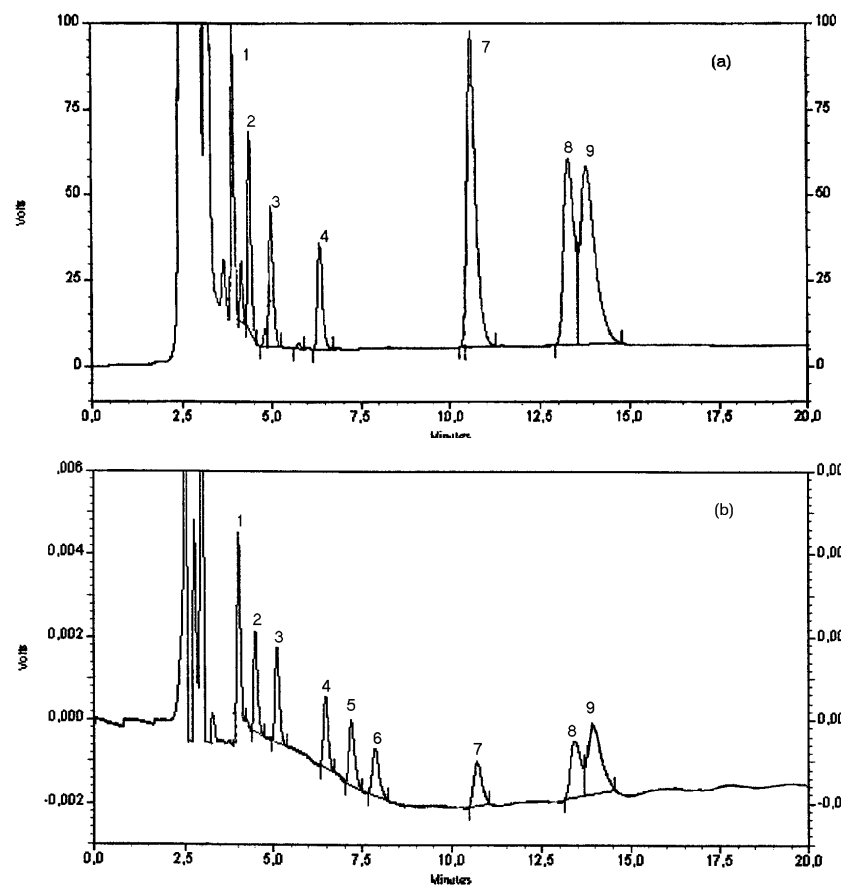

Condições cromatográficas: coluna $\mathrm{CN}(300 \times 3,9 \mathrm{~mm} ; 4 \mu \mathrm{m})$, fase móvel hexano/isopropanol (96+4), vazão 1,0mL/min.

FIGURA 2. Cromatogramas característicos dos padrões de colesterol e de óxidos de colesterol, obtidos pelo detector por conjunto de diodos fixado a $210 \mathrm{~nm}$ (a) e pelo detector por índice de refração (b). Colesterol e óxidos de colesterol numerados de acordo com a Tabela 2.

Foi realizada uma comparação entre os resultados obtidos pelo detector por conjunto de diodos e pelo índice de refração de colesterol e óxidos de colesterol identificados nas amostras de almôndegas, blanquete, peito defumado, hambúrguer, presunto, presunto defumado, roulé e salsicha (Tabela 3). Os resultados dos teores de colesterol obtidos pelos dois detectores não apresentaram diferenças significativas, apesar dos valores determinados pelo índice de refração serem maiores que os determinados pelo detector por conjunto de diodos. A separação do colesterol pelo detector por ín- dice de refração foi melhor que a separação pelo detector por conjunto de diodos, pois o colesterol eluiu mais afastado do solvente e dos interferentes presentes nas amostras (Figuras 2 e 3).
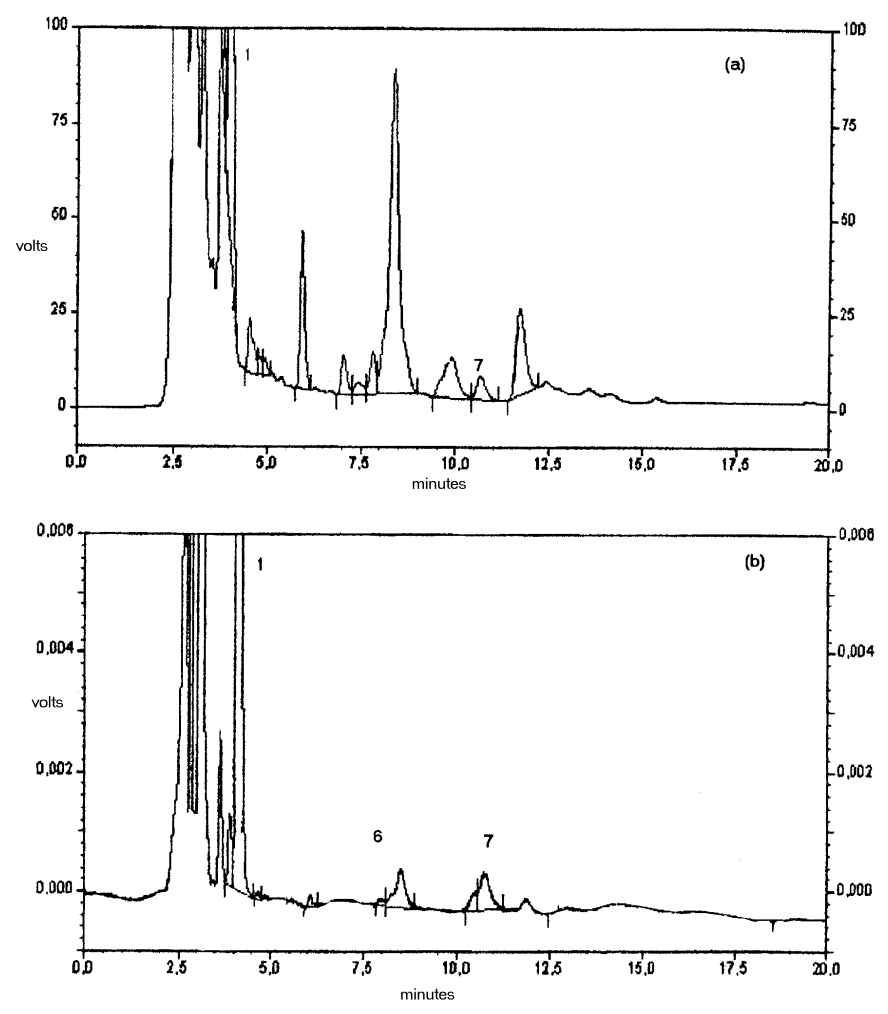

Condições cromatográficas: coluna $\mathrm{CN}(300 \times 3,9 \mathrm{~mm} ; 4 \mu \mathrm{m})$, fase móvel hexano/isopropanol (96+4), vazão $1,0 \mathrm{~mL} / \mathrm{min}$.

FIGURA 3. Cromatogramas característicos de colesterol e de óxidos de colesterol em amostra de blanquete de peru, obtidos pelo detector por conjunto de diodos fixado a 210 nm (a) e pelo detector por índice de refração (b). Colesterol e óxidos de colesterol numerados de acordo com a Tabela 2.

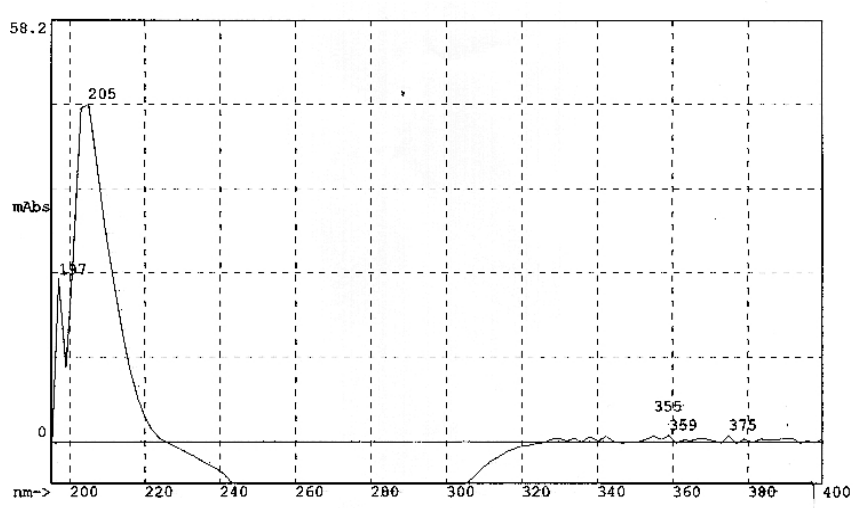

Condições cromatográficas: coluna $\mathrm{CN}(300 \times 3,9 \mathrm{~mm}$; $4 \mu \mathrm{m})$, fase móvel hexano/isopropanol (96+4), vazão 1,0mL/min.

FIGURA 4. Espectro de absorvância do colesterol da amostra blanquete de peru, obtido pelo detector por conjunto de diodos. 


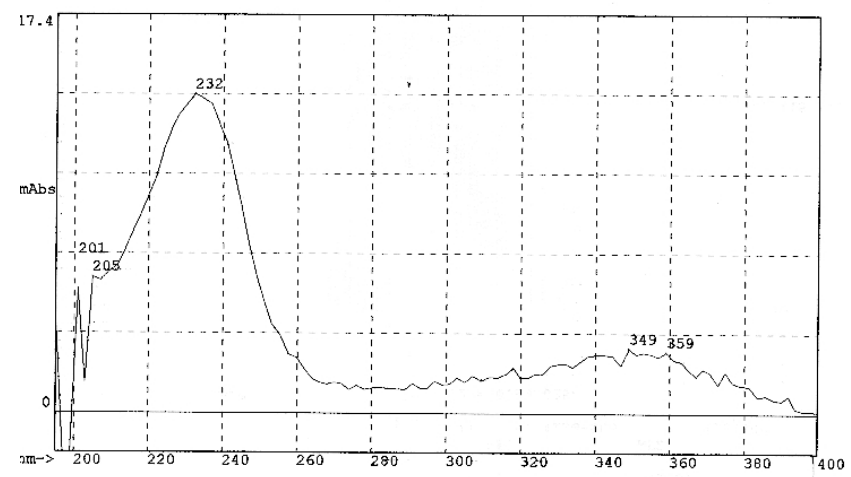

Condições cromatográficas: coluna $\mathrm{CN}(300 \times 3,9 \mathrm{~mm} ; 4 \mu \mathrm{m})$, fase móvel hexano/isopropanol (96+4), vazão $1,0 \mathrm{~mL} / \mathrm{min}$.

FIGURA 5. Espectro de absorvância do 7-cetocolesterol da amostra blanquete de peru, obtido pelo detector por conjunto de diodos.

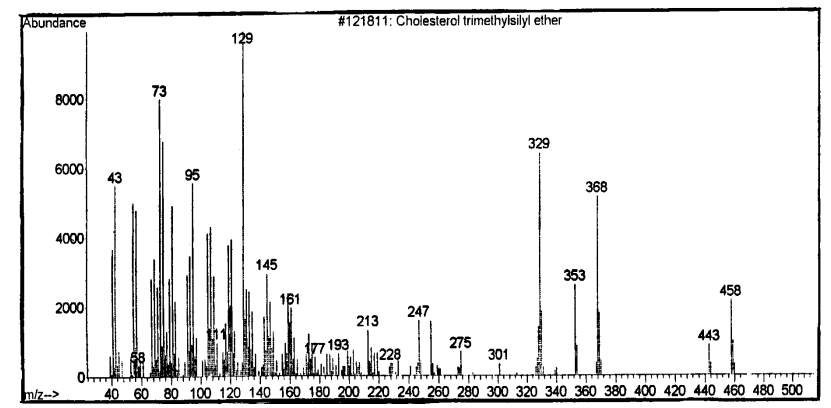

FIGURA 6. Espectro de massas do colesterol da amostra blanquete de peru, obtido por cromatografia gasosa. Energia de ionização de $70 \mathrm{eV}$. Os espectros de massas foram explorados de 40-600 (m/z).

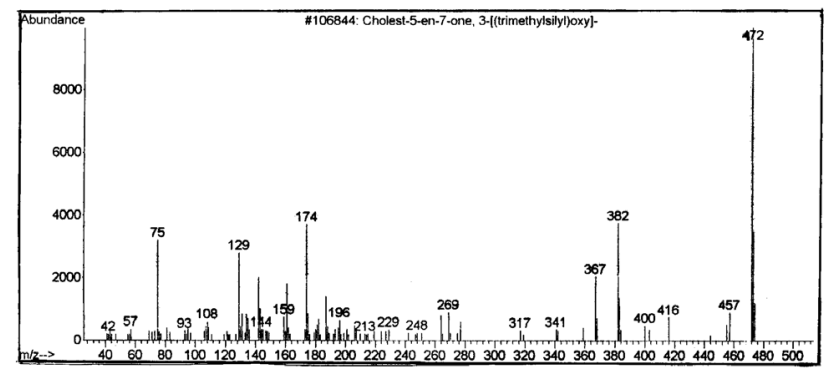

FIGURA 7. Espectro de massas do 7-cetocolesterol da amostra blanquete de peru, obtido por cromatografia gasosa. Energia de ionização de $70 \mathrm{eV}$. Os espectros de massas foram explorados de 40-600 (m/z).

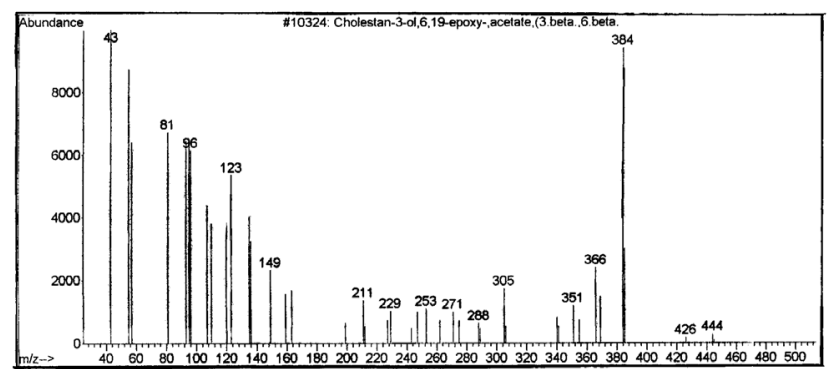

FIGURA 8. Espectro de massas do 5,6 $\beta$-epoxicolesterol da amostra blanquete de peru, obtido por cromatografia gasosa. Energia de ionização de $70 \mathrm{eV}$. Os espectros de massas foram explorados de 40-600 (m/z).

Observa-se na Tabela 3 que os valores de óxidos de colesterol variaram grandemente entre as amostras analisadas e entre os dois sistemas de detecção. O 7-cetocolesterol foi o óxido de colesterol mais freqüente entre as amostras analisadas. Tanto no detector por conjunto de diodos quanto no detector por índice de refração. No dector por conjunto de diodos os valores variaram de não detectado no blanquete, no peito defumado e no roulé a $1,84 \mu \mathrm{g} / \mathrm{g}$ na salsicha e, no índice de refração, de não detectado nas almôndegas, no blanquete, no peito defumado, no hambúrguer, no roulé e na salsicha a $17,32 \mu \mathrm{g} / \mathrm{g}$ no presunto defumado. Os valores determinados pelo índice de refração foram sempre superiores aos valores determinados pelo detector por conjunto de diodos, o que pode ser devido à presença de algum interferente.

Os teores de 5,6ß-epoxicolesterol obtidos nas amostras analisadas variaram de não detectado nas almôndegas, no blanquete, no peito defumado, no hambúrguer, no presunto e na salsicha a $4,50 \mu \mathrm{g} / \mathrm{g}$ no roulé.

TABELA 3. Comparação dos teores de colesterol (mg/100g) e 7-cetocolesterol $(\mu \mathrm{g} / \mathrm{g})$ em produtos processados de carne de peru obtidos pelos detectores por conjunto de diodos e índice de refração.

\begin{tabular}{|c|c|c|c|c|}
\hline \multirow[b]{2}{*}{ Amostras* } & \multicolumn{2}{|c|}{ Colesterol } & \multicolumn{2}{|c|}{ 7-cetocolesterol } \\
\hline & DAD & IR & DAD & IR \\
\hline \multicolumn{5}{|l|}{ Almôndegas } \\
\hline 1 & 36,0 & 40,5 & 0,50 & 2,77 \\
\hline 2 & 40,2 & 43,7 & 1,19 & 2,41 \\
\hline 3 & 39,2 & 42,1 & 0,44 & nd \\
\hline 4 & 30,0 & 33,5 & 0,65 & nd \\
\hline \multicolumn{5}{|l|}{ Blanquete } \\
\hline 1 & 35,0 & 37,8 & 0,88 & 6,96 \\
\hline 2 & 29,6 & 32,2 & 0,48 & 1,64 \\
\hline 3 & 27,3 & 28,1 & 0,51 & nd \\
\hline 4 & 25,1 & 27,7 & nd & 6,62 \\
\hline \multicolumn{5}{|c|}{ Peito defumado } \\
\hline 1 & 31,4 & 34,2 & nd & nd \\
\hline 2 & 33,3 & 40,7 & nd & nd \\
\hline 3 & 25,5 & 29,0 & 0,55 & 0,73 \\
\hline 4 & 29,5 & 32,6 & 0,35 & nd \\
\hline \multicolumn{5}{|l|}{ Hambúrguer } \\
\hline 1 & 35,7 & 38,5 & 0,82 & 9,05 \\
\hline 2 & 37,0 & 41,2 & 1,73 & nd \\
\hline 3 & 38,8 & 40,0 & 0,35 & nd \\
\hline 4 & 35,1 & 38,5 & 0,32 & 7,47 \\
\hline \multicolumn{5}{|l|}{ Presunto } \\
\hline 1 & 38,3 & 42,4 & 1,12 & 7,61 \\
\hline 2 & 38,0 & 43,1 & 0,62 & 3,16 \\
\hline 3 & 29,0 & 32,0 & 0,31 & 9,78 \\
\hline 4 & 31,8 & 35,0 & 0,44 & 5,25 \\
\hline \multicolumn{5}{|c|}{ Presunto defumado } \\
\hline 1 & 41,5 & 48,0 & 1,40 & 12,65 \\
\hline 2 & 44,4 & 53,1 & 0,47 & 17,32 \\
\hline 3 & 30,3 & 33,3 & 0,34 & 6,61 \\
\hline 4 & 35,2 & 39,0 & 0,66 & 2,37 \\
\hline \multicolumn{5}{|l|}{ Roulé } \\
\hline 1 & 42,0 & 45,2 & 1,00 & nd \\
\hline 2 & 35,8 & 37,0 & 0,38 & nd \\
\hline 3 & 28,0 & 32,1 & nd & nd \\
\hline 4 & 34,5 & 38,0 & 0,67 & 8,79 \\
\hline \multicolumn{5}{|l|}{ Salsicha } \\
\hline 1 & 37,1 & 40,2 & 1,13 & nd \\
\hline 2 & 38,5 & 41,3 & 1,84 & 9,38 \\
\hline 3 & 27,7 & 31,0 & 0,71 & 8,19 \\
\hline 4 & 29,7 & 33,1 & 0,70 & 9,12 \\
\hline
\end{tabular}


Os resultados dos testes de recuperação, limite de detecção e limite de quantificação realizados para o colesterol e para os óxidos de colesterol pelo método inteiro, ou seja, extração dos lipídios segundo FOLCH, LESS \& STANLEY [7], saponificação, extração da matéria insaponificável e determinação por CLAE utilizando detectores por conjunto de diodos e índice de refração, encontram-se nas Tabelas 4 e 5, respectivamente. Observa-se também a repetibilidade do método através do coeficiente de variação.

TABELA 4. Taxas de recuperação, limite de detecção e limite de quantificação do colesterol (mg/100g) e dos óxidos de colesterol $(\mu \mathrm{g} / \mathrm{g})$ em amostras de peito de peru defumado, determinados pelo detector por conjunto de diodos

$\begin{array}{lcccccc}\text { Compostos } & \mathrm{n} & \begin{array}{c}\text { Recuperação } \\ (\%)\end{array} & \mathrm{DP} & \% \mathrm{CV} & \begin{array}{c}\text { Limite de } \\ \text { Detecção }\end{array} & \begin{array}{c}\text { Limite de } \\ \text { Quantificação }\end{array} \\ \text { Colesterol } & 10 & 88,81 & 0,16 & 0,40 & 0,48 & 1,60 \\ \text { 7-cetocolesterol } & 10 & 91,15 & 0,03 & 0,12 & 0,09 & 0,30 \\ & & & & & \\ \begin{array}{l}\mathrm{n}=\text { número de repetições } \\ \text { DP = desvio padrão } \\ \mathrm{CV}=\text { coeficiente de variação }\end{array} & & & & & \end{array}$

TABELA 5. Taxas de recuperação, limite de detecção e limite de quantificação do colesterol (mg/100g) e dos óxidos de colesterol $(\mu \mathrm{g} / \mathrm{g})$ em amostras de peito de peru defumado, determinados pelo detector por índice de refração

\begin{tabular}{|c|c|c|c|c|c|c|}
\hline Compostos & $n$ & $\begin{array}{c}(\%) \\
\text { Recuperação }\end{array}$ & $\mathrm{DP}$ & $\% \mathrm{CV}$ & $\begin{array}{l}\text { Limite de } \\
\text { Detecção }\end{array}$ & $\begin{array}{c}\text { Limite de } \\
\text { Quantificação }\end{array}$ \\
\hline Colesterol & 10 & 90,23 & 0,24 & 0,55 & 0,72 & 2,40 \\
\hline 7-cetocolesterol & 10 & 111,44 & 0,06 & 0,25 & 0,18 & 0,60 \\
\hline $\begin{array}{l}5,6 \beta- \\
\text { epoxicolesterol }\end{array}$ & 10 & 93,90 & 0,04 & 0,30 & 0,12 & 0,40 \\
\hline
\end{tabular}

Observa-se que o resultado obtido no presente trabalho para a recuperação do 7-cetocolesterol com o detector por índice de refração $(111,44 \%)$ foi maior que o valor obtido com o detector por conjunto de diodos $(91,15 \%)$, o que pode ser devido à detecção de algum interferente. $\mathrm{O}$ valor mais elevado para a recuperação do 7-cetocolesterol com o detector por índice de refração está de acordo com os resultados obtidos nas amostras, demonstrando mais uma vez que este óxido, nas condições de análise, é melhor quantificado pelo detector por conjunto de diodos. A recuperação do 7-cetocolesterol, tanto pelo detector por conjunto de diodos $(91,15 \%)$ como pelo detector por índice de refração $(111,44 \%)$ foram maiores que os valores encontrados por CSALLANY et al. [4] com detector UV (88\%) e por NIELSEN et al. [13] utilizando detector por índice de refração (100\%).

A recuperação do colesterol pelo detector por índice de refração foi ligeiramente maior que pelo detector por conjunto de diodos. Resultados semelhantes foram obtidos na comparação entre os dois sistemas de detecção em amostras de produtos cárneos processados. A melhor separação do colesterol obtida pelo detector por índice de refração é responsável pelos valores mais elevados.

De acordo com alguns autores [4, 13] como a quantidade de óxidos de colesterol é muito pequena altas recuperações podem não ser encontradas.

Os limites de detecção do colesterol e dos óxidos de colesterol foram menores para o detector por conjunto de diodos que para o índice de refração, como esperado, uma vez que o índice de refração tem baixa sensibilidade.

O limite de detecção encontrado para o colesterol no presente trabalho, utilizando o detector por conjunto de diodos $(0,48 \mathrm{mg} / 100 \mathrm{~g})$, foi maior que o valor encontrado por CSALLANY et al. [4] $(1,10 \mu \mathrm{g} / \mathrm{g})$, enquanto que para o 7-cetocolesterol foi encontrado valor menor $(0,09 \mu \mathrm{g} / \mathrm{g})$ do que o encontrado pelos mesmos autores $(0,38 \mu \mathrm{g} / \mathrm{g})$. ROSE-SALLIN et al. [23] encontraram também valor maior para o 7-cetocolesterol, sendo $0,9 \mu \mathrm{g} / \mathrm{g}$.

A repetibilidade do método foi excelente para os dois detectores, embora o índice de refração apresentou valores maiores, provavelmente devido à dificuldade de manter o sistema de detecção estável. Os resultados obtidos são inferiores aos descritos por PENAZZI et al. [21], os quais encontraram 3,9 e 3,7\% para dois métodos utilizando CLAE. ROSE-SALLIN et al. [23] consideram que obtiveram boa repetibilidade com valores para coeficiente de variação de 0,5 a $1,2 \%$ nos óxidos encontrados (25-hidroxicolesterol, 20 $\alpha$-hidroxicolesterol, colestan-3 $\beta, 5 \alpha$-diol-6-ona, $7 \alpha$-hidroxicolesterol, $\quad 7 \beta$ hidroxicolesterol e 7-cetocolesterol).

\section{4 - CONCLUSÕES}

Através da cromatografia líquida de alta eficiência, utilizando detectores por conjunto de diodos e índice de refração foi possível quantificar com precisão o colesterol e o 5,6 $\beta$-epoxicolesterol pelo detector por conjunto de diodos, em amostras de produtos processados de carne de peru.

\section{5 - REFERÊNCIAS BIBLIOGRÁFICAS}

[1] ANSARI, G. A. S. \& SMITH, L. L. High-performance liquid chromatography of cholesterol autoxidation products. J. Chromat., v. 175, p. 307-15, 1979.

[2] CHAIRMAN, L. H. K.; CRUMMETT, W.; DEEGAN, J. J.; LIBBY, R.O.; TAYLOR, J. K. \& WENTLER, G. Principles of Environmemtal Analysis. Am. Chem. Soc., v. 55, p. 2210-2221,1983.

[3] CHEN, Y. C.; CHIU, C. P. \& CHEN, B. H. Determination of cholesterol oxides in heated lard by liquid chromatography. Food Chem., v. 50, p. 53-58, 1994.

[4] CSALlanY, A. S., KINDOM, S. E., ADDIS, P. B. \& LEE, J. HPLC method for quantitation of cholesterol and four of its major oxidation products in muscle and liver tissues. Lipids, v. 24, n. 7, p. 645-651, 1989.

[5] FINOCCHIARO, E. T. \& RICHARDSON, T. Sterol oxides in foodstuffs: a rewiew. J. Food Prot., v. 46, p. $917-$ 925, 1983. 
[6] FINOCCHIARO, E. T.; LEE, K. \& RICHARDSON, T. Identification and quantification of cholesterol oxides in grated cheese and bleached butter oil. J. Am. Oil Chem. Soc., v. 61, p. 877-882, 1984.

[7] FOLCH, J. LESS, M. \& STANLEY, S. A simple method for the isolation and purification of total lipids from animal tissues. J. Biol. Chem., v. 226, p. 497-509, 1957.

[8] GUARDIOLA, F.; CODONY, R.; ADDIS, P. B.; RAFECAS, M. \& BOATELLA, Biological effects of oxysterols: current status. Food Chem. Toxic., v. 34, n. 2, p. 193-211, 1996.

[9] HUBBARD, R. W.; ONO, Y. \& SANCHEZ, A. Atherogenic effect oxidized products of cholesterol. Prog. Food Nutr. Sci., v. 13, p. 17-44, 1989.

[10] KUMAR, N. \& SINGHAL, O. P. Cholesterol oxides and atherosclerosis: a review. J. Sci. Food Agric., v. 55, p. 497-510, 1991.

[11] MCCLUSKEY, S. \& DEVERY, R. Validation of chromatography analysis of cholesterol oxides in dried foods. Trends Food Sci. Technol., v. 4, p.175-178, 1993.

[12] MISSLER, S. R.; WAISILCHUK, B. A. \& MERRITT, C. Separation and identification of cholesterol oxidation products in dried egg preparation. J. Food Sci., v. 50, p. 595-598, 1985.

[13] NiElsen, J. H.; OlSEN, C. E.; DUEDAHL, C. \& SKIBSTED, L. H. Isolation and quantitation of cholesterol oxides in dairy products by selected ion monitoring mass spectrometry. J. Dairy Res., v. 62, p. 101-113, 1995.

[14] NOVElli, E.; ZANARDi, E.; GHIRETTI, G. P.; CAMPANINI, G.; DAZZI, G.; MADARENA, G. \& CHIZZOLINI, R. Lipid and cholesterol oxidation in frozen stored pork, salame milano and mortadella. Meat Sci., v. 48, n. 1/2, p. 29-40, 1998.

[15] NOUROOZ-ZADEH, J. Determination of the autoxidation products from free or total cholesterol: a new multistep enrichment mehodology including the enzymatic release of esterified cholesterol. J. Agric. Food Chem., v. 38, p. 1667-1675, 1990.

[16] OSADA, K.; KODAMA, T.;YAMADA, K. \& SUGANO,M. Oxidation of Cholesterol by Heating. J. Agric. Food Chem., v. 41, p. 1198-1202, 1993.

[17] PARK, S. W. \& ADDIS, P. B. Cappillary column gas liquid chromatographic resolution of oxidized cholesterol derivatives. Anal. Biochem., v. 52, p. 275283, 1985.

[18] PARK, S. W. \& ADDIS, P. B. Identification and quantitative estimation of oxidized cholesterol derivaties in heated tallow. J. Agric. Food Chem., v. 34, p. 653659, 1986a.

[19] PARK, S. W. \& ADDIS, P. B. Fuerther investigation of oxidized cholesterol derivatives in heated fats. J. Food Sci., v. 51, p. 1380- 1387, 1986b.

[20] PARK, S. W. \& ADDIS, P. B. Cholesterol oxidation products in some muscle foods. J. Food Sci., v. 52, p. 1500-1503, 1987.
[21] PENAZZI, G.; CABONI, M. F.; ZUNIN, P.; EVANGELIST, F.; TISCORNIA, E.; TOSCHI, T. G. \& LERCKER, G. Routine high-performance liquid chromatographic deternination of free 7 -ketocholesterol in some foods by two different analytical methods. J. Am. Oil Chem. Soc., v. 72, n. 12, p. 1523-1527, 1995.

[22] PENG, S. K. \& TAYLOR, C. B. Cholesterol autoxidation, health and arteriosclerosis. A review on situations in developed countries. Nutr. Diet., v. 44, p. 117-128, 1984.

[23] ROSE-SALLIN, C.; BAUMANN, E.; BUTIKOFER, U.; SIEBER, R. \& BOSSET, J. O. Contribution au dosage des oxystérols dans le lait et les produits laitiers. I. Possibilités et limites des techniques RP-HPLC. Trav. Chim. Aliment. Hyg., v. 84, p. 141-157, 1993.

[24] ROSE-SAllin, C.; HUGGeTT, A. C.; BOSSET, J. O.; TABACCHI, R. \& FAY, L. B. Quantification of cholesterol oxidation products in milk powders using $\left[{ }^{2} \mathrm{H}_{7}\right]$ cholesterol to monitor cholesterol autoxidation artifacts. J. Agric. Chem., v. 43, n. 4, p. 935-941, 1995.

[25] SANDER, B. D.; ADDIS, P. B.; PARK, S. W. \& SMITH, D. E. Quantification of cholesterol oxidation products in a variety of foods. J. Food Protect., v. 52, n. 2, p. 109-114, 1989.

[26] SARANTINOS, J.; O’DEA, K. \& SINCLAIR, A. J. Cholesterol oxides in Australian foods identification and quantitation. Food Australia. v. 45, n. 10, p. 485490, 1993.

[27] SCHMARR, H.; GROSS, H. B.; SHIBAMOTO, T. Analysis of polar cholesterol oxidation products: evaluation of a new method involving transesterification, solid phase extraction, and gas chromatography. J. Agri. Food Chem., v. 44, p. 512-517, 1996.

[28] SMITH, L. L. Cholesteol Autoxidation. Chem. Physis. Lipids, v. 44, p. 87-125, 1987.

[29] SMITH, L. L. Analysis of oxysterols by liquid chromatography. J. Liq. Chrom., v. 16, n. 8, p. 17311747, 1993.

[30] TENG, J. I.; KULIG, M. J.; SMITH, L. L.; KAN, G. \& LIER, E. V. Sterol metabolism XX. Cholesterol 7bhydroperoxide. J. Org. Chem., v. 38, p. 119-128, 1973.

[31] YAN, P. S. \& WHITE, P. J. Cholesterol oxidation in heated lard enriched with two levels of cholesterol. J. AOCS., v. 67, p. 927-935, 1990.

\section{6 - AGRADECIMENTOS}

Os autores agradecem à Fundação de Amparo à Pesquisa do Estado de São Paulo (FAPESP) e ao CNPq pelo suporte e apoio financeiro e, ao Dr. Eduardo Vicente do Centro de Química de Alimentos \& Nutrição Aplicada do Instituto de Tecnologia de Alimentos (ITAL) pela colaboração e dedicação na execução das análises com CG-MS. 